\author{
Joachim E. Fischer \\ Lucas M. Bachmann \\ Roman Jaeschke
}

\section{A readers' guide to the interpretation of diagnostic test properties: clinical example of sepsis}

Received: 30 October 2002

Accepted: 13 March 2003

Published online: 7 May 2003

(C) Springer-Verlag 2003

\author{
J. E. Fischer ( \\ Department of Pediatrics, \\ University Children's Hospital, \\ Steinweisstrasse 75, 8032 Zurich, \\ Switzerland \\ e-mail: joachim.fischer@kispi.unizh.ch \\ Tel.: +41-1-2667751 \\ Fax: +41-1-2667164 \\ L. M. Bachmann \\ Horten Centre, \\ Bolleystrasse 40 Postfach Nord, \\ 8091 Zurich, Switzerland \\ R. Jaeschke \\ Department of Medicine, \\ McMaster University, \\ Hamilton, Ontario, Canada
}

\begin{abstract}
Background: One of the most challenging practical and daily problems in intensive care medicine is the interpretation of the results from diagnostic tests. In neonatology and pediatric intensive care the early diagnosis of potentially life-threatening infections is a particularly important issue. Focus: A plethora of tests have been suggested to improve diagnostic decision making in the clinical setting of infection which is a clinical example used in this article. Several criteria that are critical to evidence-based appraisal of published data are often not adhered to during the study or in reporting. To enhance the critical appraisal on articles on diagnostic tests we discuss various measures of test accuracy: sensitivity, specificity, receiver operating characteristic curves, positive and negative predictive values, likeli-
\end{abstract}

hood ratios, pretest probability, posttest probability, and diagnostic odds ratio. Conclusions: We suggest the following minimal requirements for reporting on the diagnostic accuracy of tests: a plot of the raw data, multilevel likelihood ratios, the area under the receiver operating characteristic curve, and the cutoff yielding the highest discriminative ability. For critical appraisal it is mandatory to report confidence intervals for each of these measures. Moreover, to allow comparison to the readers' patient population authors should provide data on study population characteristics, in particular on the spectrum of diseases and illness severity.

Keywords Diagnostic tests · Sensitivity $\cdot$ Specificity $\cdot$ Receiver operating characteristic curve . Likelihood ratio $\cdot$ Infection

\section{Introduction}

Intensivists must rely on the correct interpretation of diagnostic data in a variety of clinical settings. One of the most challenging practical and daily problems in neonatology and pediatric intensive care is the diagnosis of infection [1]. Because of the consequences of delayed diagnosis $[2,3]$, physicians have low thresholds to initiate antibiotic therapy [4]. A plethora of tests has been suggested to improve diagnostic decision making in different clinical situations including sepsis, which is the example in this contribution [5, 6, 7].

To enhance the critical appraisal on articles of new diagnostic tests we discuss various concepts to measure test accuracy. A section is devoted to the importance of reporting on confidence intervals. Further problems in conducting and reporting of studies result from various sources of bias. Since these problems have been addressed in detail elsewhere, we limit the discussion to two particular issues threatening the validity of the conclusions in studies on markers of infection in neonatology and pediatric intensive care: the problem of the casecontrol design and the spectrum bias. 


\section{Prerequisites for reporting on test accuracy}

To allow determination of test accuracy a gold standard criterion must be present which allows discrimination of patients into two groups: one with infection and one without infection [8]. Ideally there should be no other difference between patients with infection and those without infection that may influence the tests results [9, 10]. The study should include all potential patients and be carried out as a cohort study [11]. Unfortunately, the reality of neonatal and pediatric intensive care enforces relevant deviations from these prerequisites. The positive blood culture does not satisfy the criterion of a gold standard since blood cultures yield false-positive and falsenegative results. Despite this fact many researchers use the positive blood culture plus clinical signs of infection as a positive gold standard, and patients without any clinical evidence plus a negative blood culture as the negative gold standard. This forces all patients to be omitted who cannot be classified unambiguously from the analysis [6]. Such analysis probably circumvents the problem of misclassification bias, at the price of introducing a new bias. Most clinicians are able to distinguish between a severely ill patient with suspected sepsis and a healthy control hospitalized in the same unit without any additional testing. Clinicians seek help from testing exactly for the ambiguous cases, which are omitted in the analysis as described above. Despite decades of research no one has as yet offered a suitable solution to this problem. The situation is much easier if an established method (e.g., blood glucose determined by the laboratory) is compared to a new method measuring the same variable (e.g., blood glucose determined by bedside tests).

\section{Measures of test accuracy}

\section{Sensitivity and specificity}

Originally $2 \times 2$ tables were defined to analyze dichotomous outcomes (e.g., death vs. survival, infected vs. noninfected) and their association with an equally dichotomous predictor variable (e.g., surfactant given vs. no surfactant, or a positive vs. a negative blood culture). Most authors still summarize test results into a $2 \times 2$ table (Fig. 1). In the situation of dichotomizing outcomes, when the test provides quantitative results, a cutoff must be chosen that distinguishes negative from positive test results. The choice of the cut-off has an important bearing on the calculated measures of test accuracy, an issue discussed below. For the moment it is assumed that an appropriate cutoff has been chosen. Once the data are tabulated, the sensitivity describes the proportion of patients with positive test results among those who are infected. The specificity denotes the proportion of patients with negative test results among those who are not in-

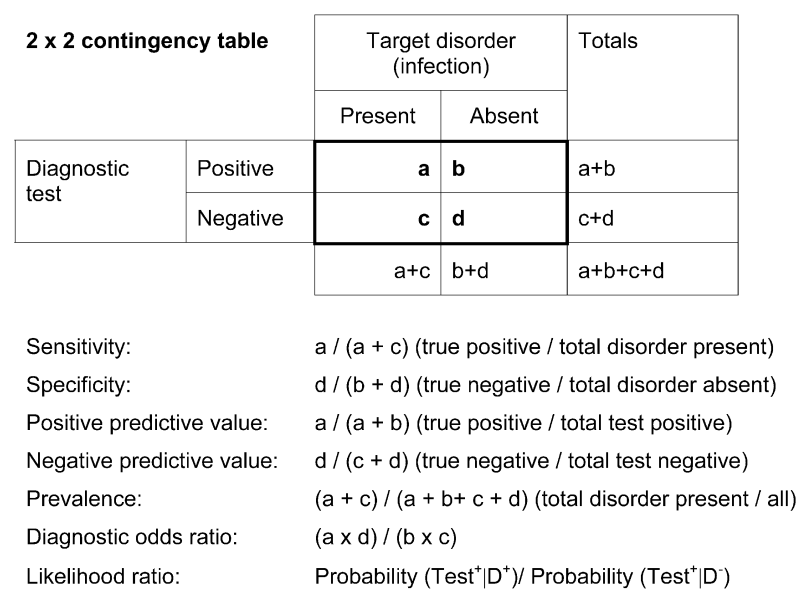

Alternative computation of the likelihood ratio in case of dichotomous outcomes: Likelihood ratio positive test: Sensitivity / (1-Specificity) Likelihood ratio negative test: (1-Sensitivity) / Specificity

\begin{tabular}{|c|c|c|c|c|c|}
\hline \multicolumn{6}{|l|}{ Example } \\
\hline \multirow{2}{*}{\multicolumn{2}{|c|}{ C-reactive protein (CRP) }} & \multicolumn{3}{|c|}{$\begin{array}{l}\text { Target disorder } \\
\quad \text { (infection) }\end{array}$} & \multirow[t]{2}{*}{ Totals } \\
\hline & & \multirow{2}{*}{$\begin{array}{r}\text { Sepsis } \\
23\end{array}$} & \multicolumn{2}{|c|}{ No sepsis } & \\
\hline \multirow{2}{*}{$\begin{array}{l}\text { Cut-off } \\
=20 \mathrm{mg} / \mathrm{l}\end{array}$} & Positive & & \multicolumn{2}{|c|}{20} & 43 \\
\hline & Negative & 3 & \multicolumn{2}{|c|}{125} & 128 \\
\hline & & 26 & 145 & & 171 \\
\hline \multicolumn{2}{|l|}{ Sensitivity: } & \multicolumn{2}{|l|}{$23 / 26$} & \multicolumn{2}{|c|}{$=0.88$, usually expressed as $88 \%$} \\
\hline \multicolumn{2}{|l|}{ Specificity: } & \multicolumn{2}{|l|}{$125 / 145$} & \multicolumn{2}{|r|}{$=0.86$, usually expressed as $86 \%$} \\
\hline \multicolumn{2}{|c|}{ Positive predictive value: } & \multicolumn{2}{|l|}{$23 / 43$} & \multicolumn{2}{|c|}{$=0.53$, usually expressed as $53 \%$} \\
\hline \multicolumn{2}{|c|}{ Negative predictive value: } & \multicolumn{2}{|l|}{$125 / 128$} & \multicolumn{2}{|r|}{$=0.98$, usually expressed as $98 \%$} \\
\hline \multicolumn{2}{|c|}{ Prevalence: } & \multicolumn{2}{|l|}{$26 / 171$} & \multicolumn{2}{|r|}{$=0.15$, usually expressed as $15 \%$} \\
\hline \multicolumn{2}{|c|}{ Likelihood ratio positive test: } & \multicolumn{2}{|l|}{$0.88 /(1-0.86)$} & \multicolumn{2}{|c|}{$=6.41$} \\
\hline \multicolumn{4}{|c|}{ Likelihood ratio negative test: $(1-0.88) / 0.86$} & \multicolumn{2}{|c|}{$=0.13$} \\
\hline \multicolumn{2}{|c|}{ Diagnostic odds ratio: } & \multicolumn{2}{|c|}{$(23 \times 125) /(20 \times 3)$} & \multicolumn{2}{|c|}{$=47.9$} \\
\hline
\end{tabular}

Fig. 1 A $2 \times 2$ contingency table for diagnostic tests. Above The calculation matrix, below a hypothetical example for plasma levels of C-reactive protein for the diagnosis of sepsis choosing a cutoff at $20 \mathrm{mg} / \mathrm{l}$ as the discrimination criterion. The prevalence was set at $15 \%$

fected. Calculation of sensitivity and specificity requires knowledge about the presence or absence of infection, determined by an independent gold standard (columns in the $2 \times 2$ table). However, in the clinical setting physicians do not know whether infection is present or absent when tests are ordered. Physicians need to make inferences about the presence or absence of infection from an obtained test result (rows in the $2 \times 2$ table). There are two ways to quantify this inference: predictive values and likelihood ratios. 
Predictive values and likelihood ratios

Likelihood ratios and predictive values provide information about the probability that a patient with a given test result is actually infected $[9,10]$. The traditional concept of predictive values (Fig. 1) presents the absolute probability that infection is present (positive predictive value) or absent (negative predictive value). Figure 2 illustrates that a major determinant of the predictive values is the prevalence of infection [12]. The same hypothetical test yields a predictive value of $85 \%$ when the prevalence is $47 \%$ but a predictive value of only $13 \%$ when the prevalence is $2.2 \%$. Thus the predictive values depend not only on the test's properties but also on the prevalence of disease in the population. Therefore they do not offer a single measure to describe the test's inherent accuracy.

To remove the difficulty arising from interpretation of predictive values decision analysts have suggested an alternative method to assess the predictive properties of a test: the likelihood ratio [10, 13, 14, 15]. Conceptually the likelihood ratio is the ratio of two probabilities, namely the probability that a specific test result is obtained in patients with the disease divided by the probability of obtaining the same test result in patients without the disease. Returning to the example provided in Fig. 1, the probability of obtaining a C-reactive protein (CRP) value exceeding $20 \mathrm{mg} / \mathrm{l}$ in patients with infection is $23 / 26$, or 0.88 . The probability of obtaining a CRP value exceeding $20 \mathrm{mg} / \mathrm{l}$ in patients without sepsis is 20/145, or 0.17 . The likelihood ratio of 6.41 is obtained by dividing the two numbers. As Fig. 2 illustrates, increasing the number of controls and thereby decreasing the prevalence does not alter the likelihood ratio. This theoretical independence from prevalence (unlike predictive values) is the first advantages of likelihood ratios. A commonsense translation of a likelihood ratio of 6.41 would be: a CRP value exceeding $20 \mathrm{mg} / \mathrm{l}$ is obtained approximately six times more often from a patient with sepsis than from a patient without sepsis. A likelihood ratio of 1 implies that the test result is equally likely to occur among patients with the disease as in patients without the disease.

In the case of dichotomous test measures, the likelihood ratios have a direct relationship to sensitivity and specificity: the likelihood ratio for a positive test result $\left(\mathrm{LHR}^{+}\right)$ could be calculated as sensitivity divided by 1 minus the specificity value. The likelihood for a negative test result $\left(\mathrm{LHR}^{-}\right)$is obtained as (1-sensitivity) divided by specificity. Figure 1 provides the mathematical equations; Fig. 3 shows a simple conversion graph for readers wanting to convert sensitivity and specificity data to likelihood ratios.

\section{Multilevel likelihood ratios}

So far we have assumed that test results are dichotomized. The disadvantage of dichotomizing is the loss of

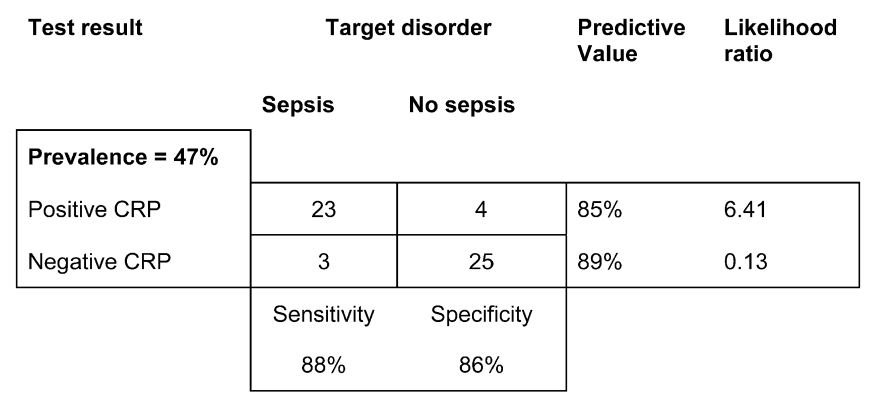

\begin{tabular}{|l|c|c|cc|}
\hline \multirow{2}{*}{$\begin{array}{l}\text { Prevalence }=\mathbf{5 1} \% \\
\text { Positive CRP }\end{array}$} & \multicolumn{5}{|c|}{} \\
\cline { 2 - 3 } Negative CRP & 23 & 20 & $53 \%$ & 6.41 \\
\cline { 2 - 3 } & 3 & 125 & $98 \%$ & 0.13 \\
\hline & Sensitivity & Specificity & & \\
$88 \%$ & $86 \%$ & & \\
& &
\end{tabular}

\begin{tabular}{|l|c|c|ll|}
\hline \multirow{2}{*}{$\begin{array}{l}\text { Prevalence }=\mathbf{5 . 2} \% \\
\text { Positive CRP }\end{array}$} & 23 & 160 & $13 \%$ & 6.41 \\
\cline { 2 - 3 } Negative CRP & 3 & 1000 & $99.7 \%$ & 0.13 \\
\cline { 2 - 3 } & Sensitivity & Specificity & & \\
$88 \%$ & $86 \%$ & & \\
& & &
\end{tabular}

Fig. 2 Dependence of the predictive values on the prevalence of infection. The example of Fig. 1 (middle) is varied to increase prevalence by reducing the number of controls in the study (above) or by increasing the numbers of controls (below). Sensitivity and specificity are held constant. As the prevalence decreases (lower two tables) the positive predictive value drops. A clinical equivalent to the lower panel would be work-up or tachypneic newborns for ruling out infection. In the lower table, a positive test result raises the probability of infection (the positive predictive value) only to $13 \%$. Unlike the predictive values, the likelihood ratio as a measure of test accuracy theoretically remains independent of prevalence. In the real world, however, the likelihood ratio may differ accross various clinical settings (e.g. due to spectrum bias, Fig. 7)

useful information. A test result may be returned from the laboratory as negative, indeterminate, or positive. Most new parameters for the diagnosis of sepsis quantitatively determine plasma compounds, with a wide range of possible results. Given the same clinical presentation, most neonatologists would consider sepsis more likely if the CRP is $130 \mathrm{mg} / \mathrm{l}$ than if it is $25 \mathrm{mg} / \mathrm{l}$. The additional information value is discarded if deliberations stop at a "positive result" (defined as plasma levels above $20 \mathrm{mg} / \mathrm{l}$ ). Figure 4 shows the example from Fig. 1 spread to a $4 \times 2$ table. The table shows that a result above $125 \mathrm{mg} / \mathrm{l}$ yields a likelihood ratio of 27.9 compared to a result between 20 and 60 , which has a likelihood ratio of 2.8. The example illustrates that reporting of multilevel likelihood ratios adds important information: it allows which levels of test results to be discerned that yield clinically important information, and which levels of test 
Fig. 3 Conversion graph for determination of likelihood ratios from sensitivity and specificity

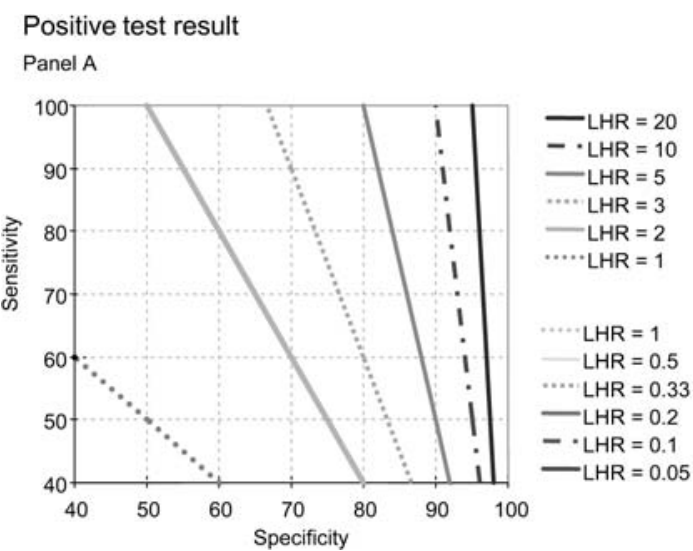

Negative test result

Panel B

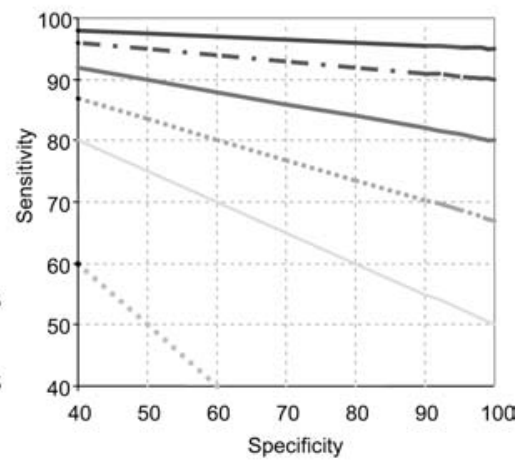

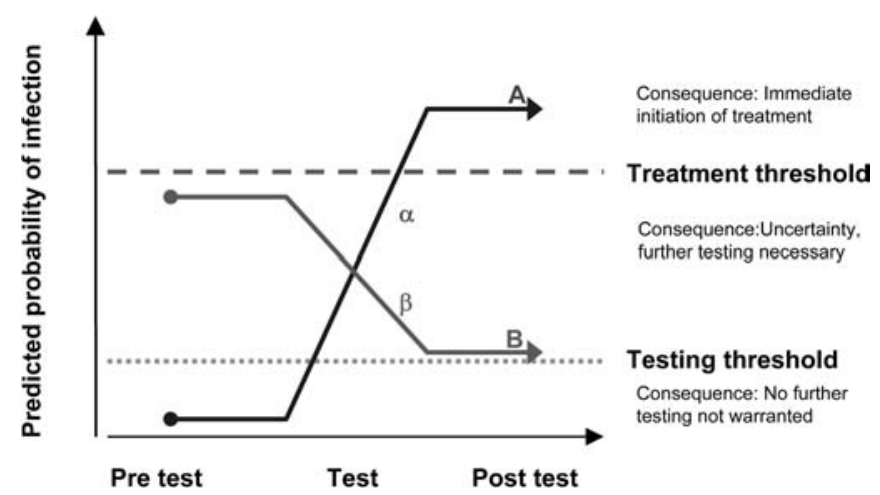

Fig. 4 Multilevel likelihood ratios. The example from Fig. 1 is expanded to a $4 \times 2$ table. The likelihood ratio differs according to the stratum of test results

results do not [9]. Unfortunately, there are no strict rules at which likelihood ratio a test result becomes clinically useful. In the present example of newborn sepsis much depends on the clinicians' prior assessment of the patient: if the level of suspicion for sepsis is already high, little additional confirmation is needed to warrant prescription of antibiotics. If the infant is deemed healthy, clinicians require much stronger evidence to alter the course of treatment. In terms of test theory, this prior judgment about the possibility that the patient has the disease is referred to as the pretest probability.

\section{Bayes' theorem}

For combining clinical judgment (the pretest probability) with a test result, likelihood ratios have an advantage over predictive values. In contrast to predictive values, likelihood ratios allow individual test results to be integrated with the physicians' judgment about the probability of infection in the patient under consideration (judgment prior to obtaining the test result). This integration is achieved by Bayesian calculations [16]. Published

\begin{tabular}{|c|c|c|c|c|c|}
\hline \multicolumn{2}{|c|}{ C-reactive protein (CRP) } & \multicolumn{2}{|c|}{$\begin{array}{l}\text { Target disorder } \\
\text { (infection) }\end{array}$} & \multirow[t]{2}{*}{ Totals } & \multirow[t]{2}{*}{$\begin{array}{l}\text { Likelihoo } \\
\text { ratio }\end{array}$} \\
\hline & & Sepsis & No sepsis & & \\
\hline \multirow[t]{4}{*}{ Plasma level } & $>125 \mathrm{mg} / \mathrm{L}$ & 10 & 2 & 12 & 27.9 \\
\hline & $61-125 \mathrm{mg} / \mathrm{L}$ & 7 & 6 & 13 & 6.5 \\
\hline & $20-60 \mathrm{mg} / \mathrm{L}$ & 6 & 12 & 18 & 2.8 \\
\hline & $<20 \mathrm{mg} / \mathrm{L}$ & 3 & 125 & 128 & 0.13 \\
\hline & & 26 & 145 & 171 & \\
\hline
\end{tabular}

Fig. 5 Probabilistic reasoning in the context of suspected infection. If infection is more likely than the probability denoted by the testing threshold, immediate initiation of antibiotics without further waiting optimizes outcomes. If the absence of infection is presumed with a certainty below the testing threshold, the risks of blood withdrawal (e.g., iatrogenic blood loss in extremely premature infants) outweighs the small risk that infection is present. Test A provides useful information by removing uncertainty (change from the pretest probability to above the treatment threshold). Test result $\mathrm{B}$ was clinically useless, because it did not sufficiently remove uncertainty. The steepness of the slopes $\alpha$ and $\beta$ corresponds to the likelihood ratio. A likelihood ratio of 1 would result in a straight horizontal line

nomograms or algorithms facilitate these computations $[14,17]$. Numerically the pretest odds are multiplied by the likelihood ratio to obtain the posttest odds. Disease odds and disease probability are related as follows: odds=probability/(1-probability). The posttest probability resulting from Bayesian computations is an individualized positive or negative predictive value. Instead of being based solely on the study prevalence, as are the predictive values, the posttest probability resulting from Bayesian calculations allows all pieces of information to be considered that are available from the individual patient and clinical situation toward determining the pretest odds or pretest probability. Useful tests generate changes from prior probability estimates to the posttest probability that alter treatment decisions [10, 14]. Figure 5 illustrates these calculations for a clinical example. However, Bayesian calculations should be used to derive the post- 


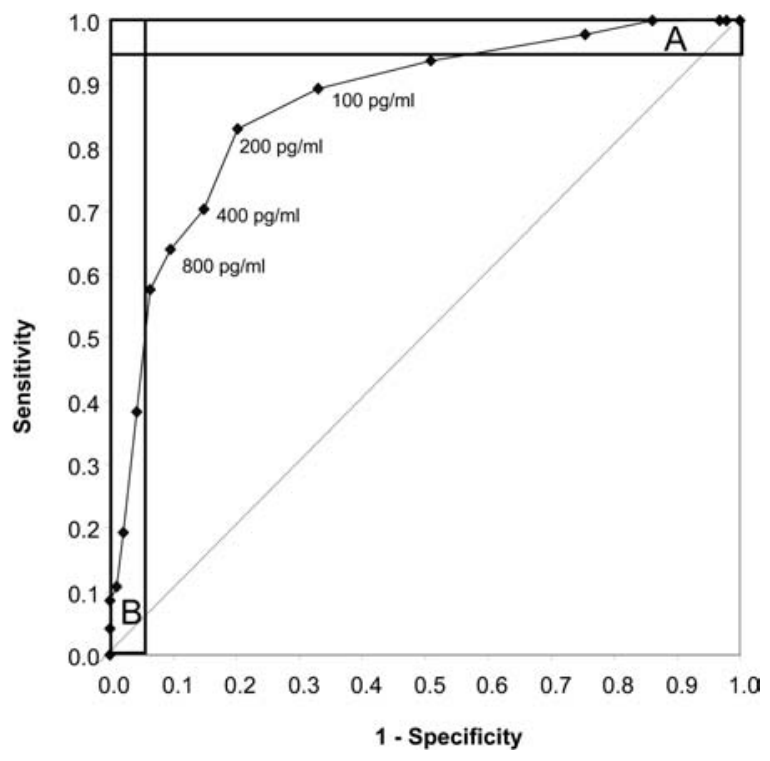

Fig. 6 Receiver operating characteristic curve. For each possible cutoff the sensitivity and specificity is determined, starting with very high values in the lower left corner down to very low cutoff values in the upper right corner (numbers in the example correspond to interleukin 6 plasma concentrations, unpublished data). The closer the curve approximates the left upper corner, the better the test. Straight dotted line Curve of a chance results (area=0.5). Accepting a margin of error of $5 \%$ (sensitivity or specificity $>95 \%$ ), cutoff values of the curve falling into rectangle $A$ are useful to rule-in infection. Values corresponding to points on the curve falling into rectangle $\mathrm{B}$ assist in ruling-out infection

test probability only when applying a single test. Bayesian chain calculations to combine multiple tests are warranted only if the tests are conditionally independent. In clinical reality test results are often correlated. A newborn found to have elevated plasma levels of interleukin8 is very likely also to show elevated levels of interleukin-6. Hence the information gain from additional measuring interleukin-6 when the interleukin- 8 result is already known is certainly less than if the interleukin-6 level were determined alone. This phenomenon is known as the conditional likelihood ratio. In practice computations become awkward, and clinicians seeking to use multiple tests to establish a diagnosis should look out for studies employing logistic regression analysis. As explained below, this type of analysis is able to consider the additional diagnostic gain of each test while considering the others simultaneously.

The receiver operating characteristic curve and the diagnostic odds ratio

A single measure that summarizes the discriminative ability of a test across the full range of cutoffs, and which is independent of prevalence is the area under the receiver operating characteristic curve (ROC). Conceptually the ROC is a plot of sensitivity against specificity for all possible cutoff values (Fig. 6). The cutoff point with the best discrimination is the point on the curve closest to the upper left corner of the graph. Areas under the curve may also be calculated for dichotomous tests, for example, the result from blood cultures. When areas under the curve are reported with standard errors or confidence intervals, they allow valuable statistical comparison of diagnostic tests $[18,19]$, particularly if applied to the same patient population as to the same diagnostic question. However, again some limitations to area comparisons require mention. Particularly for tests with intermediate to good discriminative properties (e.g., areas of $0.75-0.85$ ) the shape of the curve requires consideration. An example from newborn sepsis is the result of blood cultures. Cultures remain negative in a considerable proportion of patients in whom Gram-negative sepsis can be confirmed by histology. Thus the sensitivity is far from perfect. On the other hand, blood cultures rarely report false positive Gram-negative growth. Therefore most clinicians take a positive report of Gram-negative growth as proof. However, the area under the curve may only be around 0.7 . When interpreting curves of imperfect tests, clinicians may therefore focus on the part of interest of the curve, as marked by boxes in Fig. 6 .

An alternative way to compare tests is by means of the diagnostic odds ratio. The diagnostic odds ratio is calculated as (sensitivity $\times$ specificity)/[(1-sensitivity) $\times\left(1\right.$-specificity)] or as $\mathrm{LHR}^{+}$divided by $\mathrm{LHR}^{-}$[11] . Researchers can employ multivariate techniques to identify the cutoff with the best diagnostic odds ratio. Potentially useful tests tend to have diagnostic odds ratios well above 20 (e.g., a $\mathrm{LHR}^{+}$of 7 and a LHR ${ }^{-}$of less than 0.3 ). It can be shown mathematically that the diagnostic odds ratio is relatively independent of changes in both spectrum and prevalence. Therefore the diagnostic odds ratio provides a robust measure for dichotomous outcomes and test results. However, in the case of a test returning continuous data, the diagnostic odds ratio hinges on the chosen cutoff value.

\section{Characteristics of useful tests}

Perfect tests yield an area under the curve of 1.0. As a rule of thumb a test with an area under the curve greater than 0.9 has high accuracy, while $0.7-0.9$ indicates moderate accuracy, $0.5-0.7$ low accuracy, and 0.5 a tossup (chance result) [20]. If likelihood ratios are reported, tests with a $\mathrm{LHR}^{+}$greater than 10 or a $\mathrm{LHR}^{-}$less than 0.1 have the potential to alter clinical decisions. Tests with likelihood ratios between 5 and 10 or 0.1 and 0.2 often provide useful additional information. Tests with likelihood ratios ranging from 0.33 to 3 rarely alter clinical decisions [9]. Tests usually provide the largest gain in 
information, if the probability of disease prior to applying the test is intermediate $(>10 \%)$. In the screening mode for rare diseases, extremely good test characteristics are required to avoid large numbers of false positive results (a very high specificity).

Occasionally clinicians wish to confirm the presence or absence of infection. Positive blood cultures growing Gram-negative pathogens obtained from symptomatic patients are regarded as such proof. Because it is extremely rare to obtain positive cultures growing Gram-negative bacteria from two independent sites in patients who are not infected, a positive culture for Gram-negative pathogens has a very high specificity or a high $\mathrm{LHR}^{+}$. Although cultures remain negative in some cases of true infection (low sensitivity, poor $\mathrm{LHR}^{-}$), a positive growth of Gram-negative pathogens practically rules in sepsis.

Sometimes the clinical task is to verify the absence of infection. Almost all newborns with bacterial infection develop tachypnea or other respiratory symptoms at some stage of the infection. It is very rare to observe normal breathing patterns in newborns with infection who are left untreated. Translated into terms of test analysis, respiratory symptoms are a clinical sign with high sensitivity or a very low LHR- . However, there are many other reasons for respiratory symptoms which are not related to infection, for example, the specificity of respiratory symptoms is low and the $\mathrm{LHR}^{+}$is poor. While the absence of respiratory symptoms for more than $48 \mathrm{~h}$ after initial suspicion of infection practically rules out the disorder, the presence does not confirm infection.

\section{Considering multiple variables}

Often clinicians want to apply more than one test [21]. Unfortunately, results from different tests are usually not independent of each other. Patients with elevated procalcitonin levels often also have elevated interleukin-8 levels. Neutropenic newborns with sepsis are more likely to present with thrombocytopenia [21]. As noted above, the lack of independence renders it inappropriate to perform simple sequential Bayesian calculations. More sophisticated statistical methods must be employed that control for interdependence or colinearity [22]. This is achieved by multivariable logistic regression analysis [23]. Conceptually such analysis estimates the predicted probability that infection is present, simultaneously considering more than one variable. Multivariable regression analysis not only controls for potential interdependence of variables but also allows additional variables to be considered that may confound the results. For example, premature infants may respond with less pronounced increases in cytokine levels than term newborns. On the other hand, premature infants may be more likely to be infected than term newborns. An analysis not controlling for the effect of gestational age may underestimate the diagnostic accuracy of a parameter.
Logistic regression analysis provides regression coefficients for each variable. Positive regression coefficients and confidence intervals that do not include zero indicate that a higher level of the variable or the parameter is associated with an increased probability of infection. Confidence intervals that include zero imply that the variable does not significantly contribute to the prediction. The regression coefficients also allow cumulative diagnostic information to be determined when more than one variable is considered. An example is the Pediatric Risk of Mortality III score for predicting mortality. The score points, which are assigned to each criterion, were derived directly from the regression coefficients.

A limitation to entering multiple variables into the regression analysis is the requirement for sample size. If fewer than ten cases of infection are available per variable entered into the model, regression coefficients tend to become imprecise. An accepted practice to evaluate regression models is to divide the whole dataset into a derivation dataset (from which the prediction model is developed) and a smaller validation dataset in which the models predictive performance is tested [23].

\section{Confidence intervals}

It should be mandatory to report confidence intervals for any measure of diagnostic accuracy, including regression coefficients in multivariable regression models. The lower and upper limits of the $95 \%$ confidence intervals inform the reader about the interval in which $95 \%$ of all estimates of the measure (e.g., sensitivity, likelihood ratio or area under the curve) would fall if the study was repeated over and over again. This somewhat tricky, but mathematically correct definition, should be interpreted as following: If there is no bias distorting the observed data, it is also likely that the true population parameter lies between the lower and upper limit of the 95\% confidence interval with a probability of $95 \%$. If bias is present, the true population parameter may lie anywhere (for example, if someone measured the blood pressure in 1000 infants using the wrong cuff size, the true arterial pressure of the general population of similar infants may well lie outside the obtained narrow confidence interval).

When likelihood ratios are reported, confidence intervals that include 1 indicate that the study has not shown statistically convincing evidence of any diagnostic value of the investigated parameter. Therefore the reader does not know whether a test with a $\mathrm{LHR}^{+}$of 18 and a $95 \%$ confidence interval of $0.8-85$ is useful. A study reporting a $\mathrm{LHR}^{+}$of 5.2 with a $95 \%$ confidence interval of 4.6 -6.0 certainly provides more precise evidence than another study arriving at a $\mathrm{LHR}^{+}$of 9 with a $95 \%$ confidence interval of $2-19$.

Narrow confidence intervals imply that a very large number of patients with and without disease were ob- 
served. If the number of patients with and without disease differs widely, the width of the confidence interval hinges on the smaller group. Usually the sample size in pediatric studies is small, leading the very wide confidence intervals. Likewise, most of the studies in newborns or critically ill children are underpowered to allow statistically sound inferences about the differences in test accuracy. The invalidity of drawing conclusions from an area under the curve of 0.83 compared to an area of 0.80 becomes immediately apparent when confidence intervals are reported such as: 0.83 (95\% CI $0.69-0.96)$ vs. $0.80(95 \%$ CI $0.66-0.94)$.

\section{Sources of systematic biases}

Several important source of bias exist that may lead to overestimation of the test accuracy. First, due to the difficulty to adjudicate on the presence or absence of infection in all patients most studies on diagnostic markers of infection rely on undisputable definitions of cases (infected patients) and controls (uninfected patients), while all episodes with potentially ambiguous classification are omitted from the analysis. The consequence is a casecontrol design. In a recent meta-analysis investigators assessed the influence of various features of the study design on the diagnostic odds ratio. They identified the case-control design as the most important source of bias for overestimating test accuracy (relative diagnostic odds ratio $3.0 ; 95 \%$ confidence interval, 2.0-4.5). Deviation from other desirable features of the ideal study on diagnostic tests, for example, the lack of blinding, had less bearing on the estimates of the diagnostic accuracy [11]. The effect of the case-control study bias on more familiar terms of test accuracy is illustrated in the following example: Assuming a true likelihood ratio of a test of 5.7 for a positive result and of 0.17 for a negative result (e.g., specificity and sensitivity of $85 \%$, respectively), according to the analysis by Lijmer and coworkers [11], the case-control design bias might inflate the likelihood ratio to 10 for a positive result and to 0.1 for a negative result (e.g., sensitivity and specificity of $91 \%$ ).

Having considered the above points regarding reporting of test accuracy study, readers want to know in the end whether the study data are applicable to their own patients. This is the time to critically ask: did the study investigate a similar spectrum of severity of disease as well as potential differential diagnoses as encountered in the readers setting? It is a prudent question to ask, since any of the patient characteristics related to severity of illness, comorbidity or closely related pathologies may affect the results of the tests. Returning to the example of CRP measurements, most intensivists share the observation that patients who are more seriously ill tend to yield higher plasma levels. The consequence is that the test appears more sensitive in very ill patients than in the less

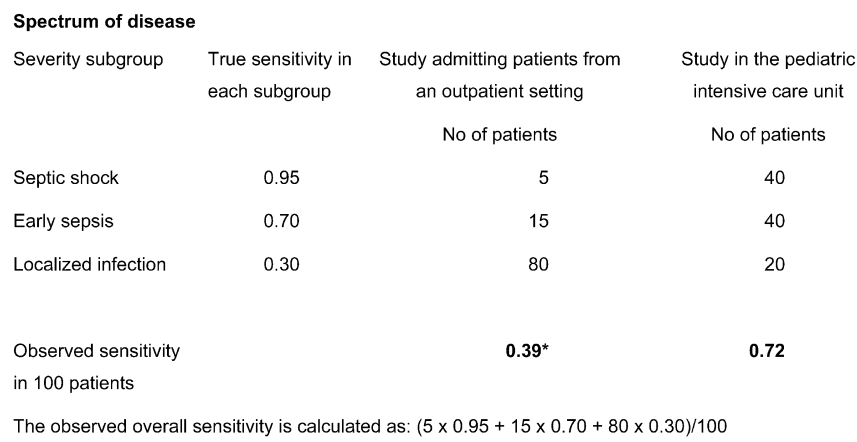

Spectrum of alternative diagnoses

Alternative condition True specificity in

(controls) subgroup

Study in the neonatal

intensive care unit

Study in the pediatric intensive care

No of controls enrolled

No of controls enrolled

Post-surgical

0.50

inflammatory

response (SIRS)

Viral infection

0.70

5

Healthy

0.99

20

Observed specificity

0.98

0.64

in 100 patients

Fig. 7 Spectrum bias. Above The influence of disease severity. Plasma levels of cytokines in patients with sepsis tend to be increase with severity. Thus for different strata of illness severity the sensitivity differs (if the cutoff is held constant). Therefore the overall sensitivity in the study population depends on the case-mix of severity, as shown in columns 2 and 3. Below The influence of disease spectrum. If the control population contains few patients, who show elevated cytokine concentrations (e.g., after surgery), the specificity is high. If the list of differential diagnoses to be considered includes conditions that are associated with positive test results, specificity drops. Holding all other factors constant, either effect alone can profoundly change likelihood ratios, the area under the receiver operating curve, and the diagnostic odds ratio

severely ill (e.g., at a cutoff of $20 \mathrm{mg} / \mathrm{l}$ ). This propensity is illustrated in the upper panel of Fig. 7 (first row) as a descending sensitivity with decreasing illness severity. Assuming that two investigators conduct two studies on the sensitivity of CRP, one in an outpatient emergency department, the other in the pediatric intensive care unit of the same hospital, and both admit 100 consecutive cases, the reported results are likely to differ, as illustrated by the second and third row of the upper panel. The reader must decide which population does more closely matches the own mix of illness severity.

Unfortunately, spectrum bias affects not only sensitivity. The lower panel of Fig. 7 presents the specificity of CRP with various differential diagnosis of sepsis. Consider a third study being conducted in the neonatal intensive care unit of the hospital. Because physicians do not admit patients after surgery to this unit, the differential diagnosis "postsurgical inflammation" is virtually absent 
from the list of alternative diagnoses. The lack of other possible cause for CRP elevation boosts specificity (second row), unlike in the pediatric intensive care unit (third row) where postsurgical inflammation is common. In summary, the mix of severity of illness is likely to affect sensitivity, while the mix of alternative diagnosis may affect specificity. Moreover, if sensitivity and specificity are affected by case mix and severity, so is the likelihood ratio. To allow critical appraisal of the applicability of a report, authors should therefore be required to provide sufficient data on patients who were not included as well as indicators for severity of disease, case mix, and other potential sources of spectrum bias.

\section{Summary}

In conclusion, articles on the diagnostic accuracy of new tests should provide the following technical information: a plot of the raw data, multilevel likelihood ratios, a plot showing the relationship between sensitivity and specificity for various cutoff levels (the receiver operating characteristic curve) and a computation of the area under the curve. All measures should be reported with confidence intervals. To allow comparison to more traditional reports, the sensitivity and specificity for the cutoff yielding the highest discriminative ability (defined as the cutoff point on the receiver operating characteristic curve closest to the upper left corner of the graph) should be reported. This review discusses the assessment of test accuracy in the context of quantitative tests to aid decision making in pediatric patients with suspected infection. The principles laid out in this overview can also be applied to the interpretation of clinical symptoms and nonlaboratory tests (e.g., the value of computed tomography scans for the diagnosis of appendicitis).
The criteria for assessing studies on diagnostic tests in infection can be summarized in the following set of question that can be put to individual studies [18]:

- Criteria related to conduction and report of the study

- Were the criteria for outcome adjudication (infected vs. not infected) adequately described to permit their replication?

- Was the outcome assessed blind to the results of the test under investigation?

- Did the results of the test being evaluated influence the decision to perform the reference standard (e.g., obtaining blood cultures)?

- Has the diagnostic test been evaluated in a patient sample that included an appropriate spectrum of disease severity, treated and untreated patients and individuals with disorders considered as differential diagnosis?

- Was the sample obtained at the time when a decision had to be made?

- Were the inclusion and exclusion criteria for patients adequately described?

- Was the reproducibility of the test result (precision) described?

- Criteria related to the applicability of the study to concurrent patients

- Have the methods for carrying out the test been described (including sampling techniques) detailed enough to permit their exact replication?

- Would the results change the management of the patients?

- Will a patient be better off as a result of the test?

- Criteria related to the technical report of test accuracy

- Was a plot of the raw data provided?

\section{References}

1. Escobar GJ (1999) The neonatal "sepsis work-up": personal reflections on the development of an evidencebased approach toward newborn infections in a managed care organization. Pediatrics 103:360-373

2. Stoll BJ, Gordon T, Korones SB, Shankaran S, Tyson JE, Bauer CR, Fanaroff AA, Lemons JA, Donovan EF, Oh W, Stevenson DK, Ehrenkranz RA, Papile LA, Verter J, Wright LL (1996) Early-onset sepsis in very low birth weight neonates: a report from the National Institute of Child Health and Human Development Neonatal Research Network. J Pediatr 129:72-80
3. Benitz WE, Gould JB, Druzin ML (1999) Preventing early-onset group B streptococcal sepsis: strategy development using decision analysis. Pediatrics 103:e76

4. Hammerschlag MR, Klein JO, Herschel M, Chen FC, Fermin R (1977) Patterns of use of antibiotics in two newborn nurseries. N Engl J Med 296:1268-1269

5. Berner R, Niemeyer C, Leititis J, al. e (1998) Plasma levels and gene expression of granulocyte colonystimulating factor, tumor necrosis factor-a, interleukin (IL) 1-b, IL-6, IL-8, and soluble intercellular adhesion molecule- 1 in neonatal early onset sepsis. Pediatr Res 44:469-477
6. Kuster H, Weiss M, Willeitner AE, Detlefsen S, Jeremias I, Zbojan J, Geiger R, Lipowsky G, Simbruner G (1998) Interleukin-1 receptor antagonist and interleukin-6 for early diagnosis of neonatal sepsis 2 days before clinical manifestation. Lancet 352:1271-1277

7. Chiesa C, Panero A, Rossi N, Stegagno M, De Giusti M, Osborn JF, Pacifico L (1998) Reliability of procalcitonin concentrations for the diagnosis of sepsis in critically ill neonates. Clin Infect Dis 26:664-672 
8. Isaacman DJ, Karasic RB, Reynolds EA, Kost SI (1996) Effect of number of blood cultures and volume of blood on detection of bacteremia in children. J Pediatr 128:190-195

9. Jaeschke R, Guyatt GH, Sackett DL (1994) Users' guides to the medical literature. III. How to use an article about a diagnostic test. B. What are the results and will they help me in caring for my patients? The Evidence-Based Medicine Working Group. JAMA 271:703-707

10. Jaeschke R, Guyatt G, Sackett DL (1994) Users' guides to the medical literature. III. How to use an article about a diagnostic test. A. Are the results of the study valid? EvidenceBased Medicine Working Group. JAMA 271:389-391

11. Lijmer JG, Mol BW, Heisterkamp S, Bonsel GJ, Prins MH, van der Meulen JH, Bossuyt PM (1999) Empirical evidence of design-related bias in studies of diagnostic tests. JAMA 282:1061-1066
12. Smith JE, Winkler RL, Fryback DG (2000) The first positive: computing positive predictive value at the extremes. Ann Intern Med 132:804-809

13. Dujardin B, Van den Ende J, Van Gompel A, Unger JP, Van der Stuyft P (1994) Likelihood ratios: a real improvement for clinical decision making? Eur J Epidemiol 10:29-36

14. Pauker SG, Kassirer JP (1980) The threshold approach to clinical decision making. N Engl J Med 302:1109-1117

15. Weinstein MC, Fineberg HV (1980) Clinical decision analysis. Saunders, Philadelphia

16. Pauker SG, Kopelman RI (1992) Interpreting hoofbeats: can Bayes help clear the haze? N Engl J Med 327:1009-1013

17. Fagan TJ (1975) Nomogram for Bayes theorem. N Engl J Med 293:257

18. Hanley JA, McNeil BJ (1983) A method of comparing the areas under receiver operating characteristic curves derived from the same cases. Radiology 148:839-843

19. McNeil BJ, Hanley JA, Funkenstein HH, Wallman J (1983) Paired receiver operating characteristic curves and the effect of history on radiographic interpretation. CT of the head as a case study. Radiology 149:75-77
20. Swets JA (1988) Measuring the accuracy of diagnostic systems. Science 240:1285-1293

21. Mahieu LM, De Muynck AO, De Dooy JJ, Laroche SM, Van Acker KJ (2000) Prediction of nosocomial sepsis in neonates by means of a computerweighted bedside scoring system (NOSEP score). Crit Care Med 28:2026-2033

22. Tosteson AN, Weinstein MC, Wittenberg J, Begg CB (1994) ROC curve regression analysis: the use of ordinal regression models for diagnostic test assessment. Environ Health Perspect 102 [Suppl 8]:73-78

23. Bagley SC, White H, Golomb BA (2001) Logistic regression in the medical literature: standards for use and reporting, with particular attention to one medical domain. J Clin Epidemiol 54:979-985 